\title{
Efficacy and safety of nintedanib in patients with systemic sclerosis-associated interstitial lung disease treated with mycophenolate: A subgroup analysis of the SENSCIS trial
}

Interstitial lung disease (ILD) in scleroderma patients contributes to the mortality of the disease. Immunosuppression with mycophenolate has become the mainstay of treatment to limit the decline in forced vital capacity (FVC), one of the main predictors of mortality in these patients.

The Safety and Efficacy of Nintedanib in Systemic Sclerosis (SENSCIS) trial was a randomised, double-blind, placebo-controlled, parallel-group, phase 3 trial. It included participants from 32 countries with scleroderma-associated ILD with at least 10\% fibrosis, FVC\% predicted of at least $40 \%$, and diffusion capacity of more than $30 \%$. The participants were randomised to receive nintedanib or placebo for 52 weeks.

In this post-hoc subgroup analysis, patients on mycophenolate at baseline were assessed for the annual rate of decline in FVC ( $\mathrm{mL}$ per year) and absolute change from baseline FVC $(\mathrm{mL})$. The minimal important difference was a change in $\mathrm{FVC}$ of $3.3 \%$ predicted at week 52. Secondary endpoints included clinical effects with the modified Rodnan skin score (mRSS) or health-related quality of life measured using the St George's Respiratory Questionnaire (SGRQ). Safety and adverse events were also assessed, which were similar across all groups of mycophenolate use, with gastrointestinal adverse effects being the most common adverse event leading to discontinuation of treatment.

A total of 580 patients were randomised into the nintedanib and placebo groups and further subdivided into groups who were on mycophenolate at baseline or not.

At baseline, 139 (48\%) patients in the nintedanib group were receiving mycophenolate and 140 (49\%) were in the placebo group. There was a higher proportion of patients with diffuse cutaneous sclerosis, with worse mRSS scores and lower mean FVC\% predicted in the mycophenolate group compared with those not taking mycophenolate.

Compared with the placebo, nintedanib reduced the annual rate of decline in FVC in both patients taking mycophenolate at baseline and those not taking mycophenolate, although the treatment effect was smaller in those taking mycophenolate, with mean differences of $26.3 \mathrm{~mL} /$ year and $55.4 \mathrm{~mL} /$ year in those taking mycophenolate and in those not taking mycophenolate, respectively. The annual rate of decline in FVC in participants taking mycophenolate at baseline who were treated with nintedanib was $40.2 \mathrm{~mL}$ per year (standard deviation (SD) 19.8), close to the annual rate of decline in FVC observed in healthy adults. In the group receiving nintedanib at baseline, the mean (SD) FVC declined by 66.5 (19.3) $\mathrm{mL}$ per year and in those only receiving placebo, it declined by 119.3 (19.0) $\mathrm{mL}$ per year.

Lung function decline, as assessed by an absolute decrease in FVC of at least $3.3 \%$ predicted, was lower with nintedanib than placebo in those taking mycophenolate at baseline, $29 \%$ v. $40 \%$ (odds ratio 0.61 (95\% confidence interval 0.37 - 1.01)).

This suggests that combining immunosuppression with an antifibrotic such as nintedanib, might provide additional benefits in reducing the progression of scleroderma interstitial lung disease. Further trials are needed to assess the benefits of initial combination therapy $\mathrm{v}$. the individual drugs or even sequential treatment.

\section{Elizabeth Louw}

Division of Pulmonology, Department of Medicine, Stellenbosch

University, Cape Town, South Africa

ehdutoit@googlemail.com 